\title{
A microcomputer interface for applications requiring large numbers of subjects
}

\author{
LLOYD HASTINGS and N. DEREK ARNOLD \\ Department of Environmental Health, College of Medicine \\ University of Cincinnati, Cincinnati, Ohio 45267
}

\begin{abstract}
While most small microcomputers are powerful enough to control intricate experiments, a major limitation of many is their limited I/O capability. An interface for the KIM-1 or similar small microcomputer is described that provides for up to 128 or more inputs. The basic components of the interface are a series of shift registers through which the incoming data are manipulated and read by the microcomputer. Provisions are also made to latch the incoming data, allowing detection of very high rates of or even simultaneous data input. The interface is modular in design and can be expanded by increments of eight inputs. Use of the system to monitor a large number of running wheels is discussed.
\end{abstract}

The development of the microcomputer has provided the research psychologist with a very powerful and efficient way to conduct experiments. It is truly amazing that an instrument costing only several hundred dollars has the capability to monitor a multitude of events and respond accordingly on a real-time basis. However, a serious limitation with many microcomputers is their limited capacity to interface with the real world, that is, a paucity of input/output (I/O) lines. Most small, singleboard microcomputers, such as the KIM-1, AIM, and so on, have $16 \mathrm{I} / \mathrm{O}$ lines as a standard feature. While this is adequate for many applications, most psychological experiments require more. This is especially true in studies using operant conditioning procedures in which many events must be monitored. Similarly, in tasks in which only a few parameters are monitored, such as with activity measures, it is usually necessary to run a large number of subjects. This requires a large number of $\mathrm{I} / \mathrm{O}$ lines. Thus the requirements of such studies far exceed the capacity of these microcomputers.

This problem can be resolved in two ways. The first solution is to expand the number of $I / O$ lines available; this is normally accomplished by adding additional I/O chips (Osaka, 1980). While this provides the necessary expansion, it also results in increased execution time, since each line must be addressed and then examined. If not too many additional $\mathrm{I} / \mathrm{O}$ lines are required, this may be a feasible and cost-effective solution.

The alternate method of increasing $\mathrm{I} / \mathrm{O}$ capacity is to encode the incoming lines before they reach the $\mathrm{I} / \mathrm{O}$ ports. Theoretically, an 8-bit port would allow 256 discrete devices to be addressed. However, the number of discrete components necessary to translate the 8-bit address into a single device would be large (but certainly practicable). The following is a description of an interface system that employs a variation of this approach but needs only 3 bits of an 8-bit port to monitor up to 128 inputs. Furthermore, the system is modular in design, allowing additional lines to be added eight at a time and as needed.

A major advantage of this system is that the data are latched until the computer can read them. This means that many events can occur simultaneously and all will be detected by the computer. This eliminates the need of trying to select the duration for a oneshot that is triggered by an external event that is not so short that it will terminate before the computer can detect it or so long that it will mask others. One limitation of this system is that it is configured to handle inputs only; an additional system is now under development that will handle both.

This system was originally designed to monitor 48 running wheels using a KIM-1 microcomputer. Some method was needed to record and store the number of wheel revolutions produced by a rat on an hourly basis. The system had to operate $24 \mathrm{~h} /$ day, 7 days/week, for periods up to 3 months.

The I/O system as finally implemented consists of two major components: (1) individual $I / O$ cards, each capable of receiving up to eight inputs, and (2) a motherboard, into which the $1 / 0$ cards are inserted. Software controls the function of the interface. Although the software was written for the KIM-1, changing the address of the $\mathrm{I} / \mathrm{O}$ port allows it to run on any 6502based machine. The algorithm can be easily adapted to any other microprocessor. The interface can be used in any application that involves a large number of inputs.

\section{DESCRIPTION OF INDIVIDUAL INTERFACE CARD}

Each interface card has five integrated circuits, 24 resistors, and 16 capacitors. Each card is capable of interfacing eight inputs to the microcomputer. Cards are stackable; thus two cards equal 16 inputs, three cards equal 24 inputs, and so on. Stacking cards does not 
require any more lines to the computer than does singlecard operation. Cards are made as plug-in modules to simplify repair and interchangeability.

\section{CARD OPERATION}

The interface card is a simple device consisting of two blocks. One block consists of eight memory cells, made up of two 74LS279 flip-flops. The other block is an eight-channel-to-one multiplexer made up of one 74164 and two 7403s (see Figure 1). The memory cells debounce and latch each input. Under control of the computer, the multiplexer sequentially places the output of the memory cells onto the data line. Also, as the multiplexer changes from one memory cell to the next, the cell just read is cleared by the multiplexer, allowing new data to be latched in.

\section{MEMORY CELL OPERATION}

Each memory cell is composed of an R-S NAND gate flip-flop (Figure 2). A 74LS279 has four flipflops in a single package. Two of these chips are placed on each card for a total of eight memory cells on each card. Each input of the flip-flop is made edge sensitive with R-C networks. The $S$ input of the flip-flop is used for data input. The $\mathrm{R}$ input of the flip-flop is connected to the multiplexer read line so that, as the read line is turned off, the cell resets itself. Upon testing the cards with standard 7400-series logic devices, we found that the positive spike from the rising edge of the data input prematurely reset the memory cell. To avoid this and to

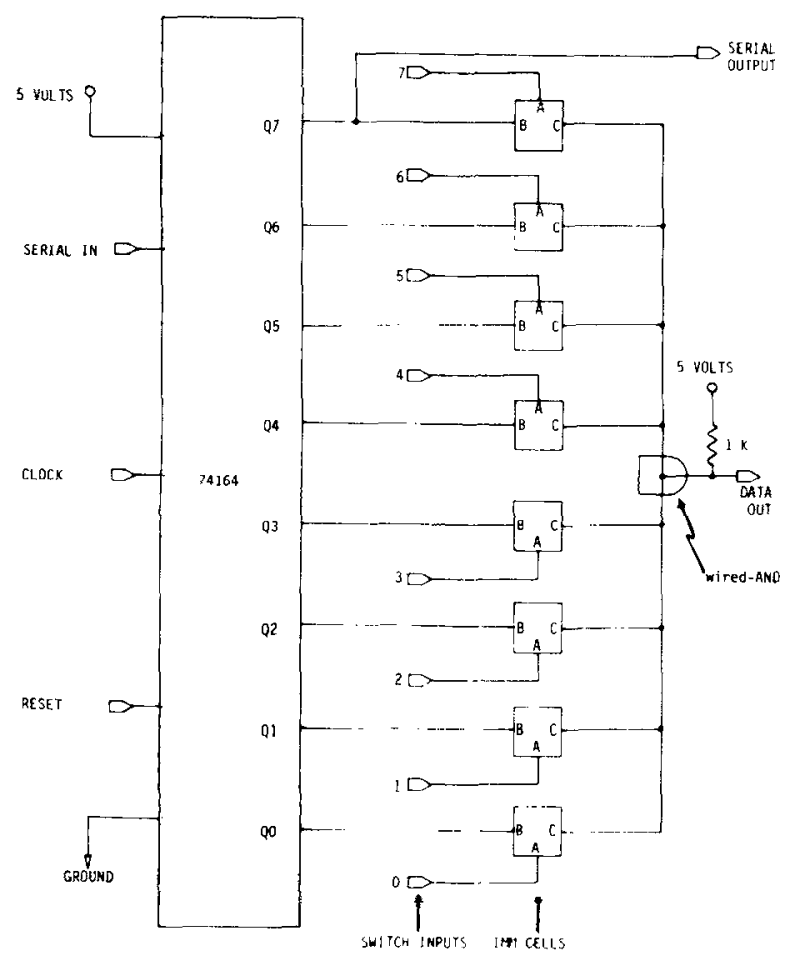

Figure 1. Functional block diagram of interface card circuitry.

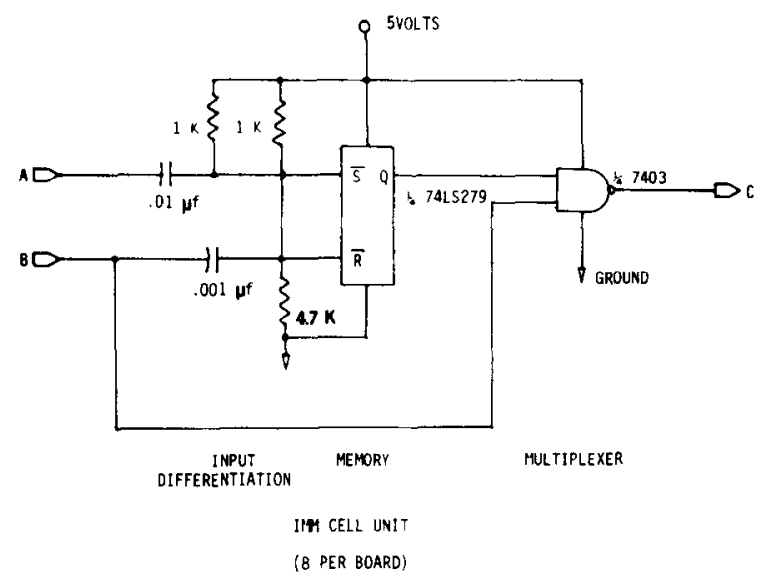

Figure 2. Circuit diagram of an input memory module (IMM).

eliminate the need for diode clamping on each input, the $74 \mathrm{LSOO}$ series was used.

\section{MULTIPLEXER OPERATION}

The multiplexer is composed of three ICs, a 74164 shift register, and two 7403 quad NAND gates with open collector outputs. The 74164 is a serial input/ parallel output shift register. The eight outputs of this register are the basis for eight inputs per card. The shift register on each card is cleared on start-up via a front panel switch called "master reset." Each output of the register is tied to one input of a NAND gate on the 7403s. The other input of the NAND gate is tied to the output of the memory cell.

In the initial state of the card, each output of the register is low and is fed to one input of a NAND gate. Since every NAND gate has at least one low input (from the shift register), all the NAND gates have high outputs. All of the outputs from the NAND gates are wired together in the "wired AND" configuration. With all NAND gates outputs high, the card data line is "ANDED" high. To read each memory cell, a single high is clocked serially from $Q_{A}-Q_{H}$ of the shift register. The $Q$ outputs that are low create a high output on the corresponding NAND gate output. The single high $Q$ output allows the selected NAND gate to bring the data line low if the memory cell is high and high if the memory cell is low (data inversion). As the high is clocked sequentially through each $Q$ output, inverted data appear at the data output sequentially starting with $Q_{A}$ 's associated memory cell and ending with $\mathrm{Q}_{\mathrm{H}}$ 's associated memory cell. The falling edge of each $Q$ resets the corresponding memory cell. $Q_{H}$ is brought out on a separate line and becomes the serial input for the next card. The algorithm for the entire operation is shown in Figure 3.

\section{MOTHERBOARD CIRCUTTRY}

The design of the motherboard is for six cards, giving 48 inputs. Two DB-25S connectors are used as the input connectors. Each memory cell input is connected 


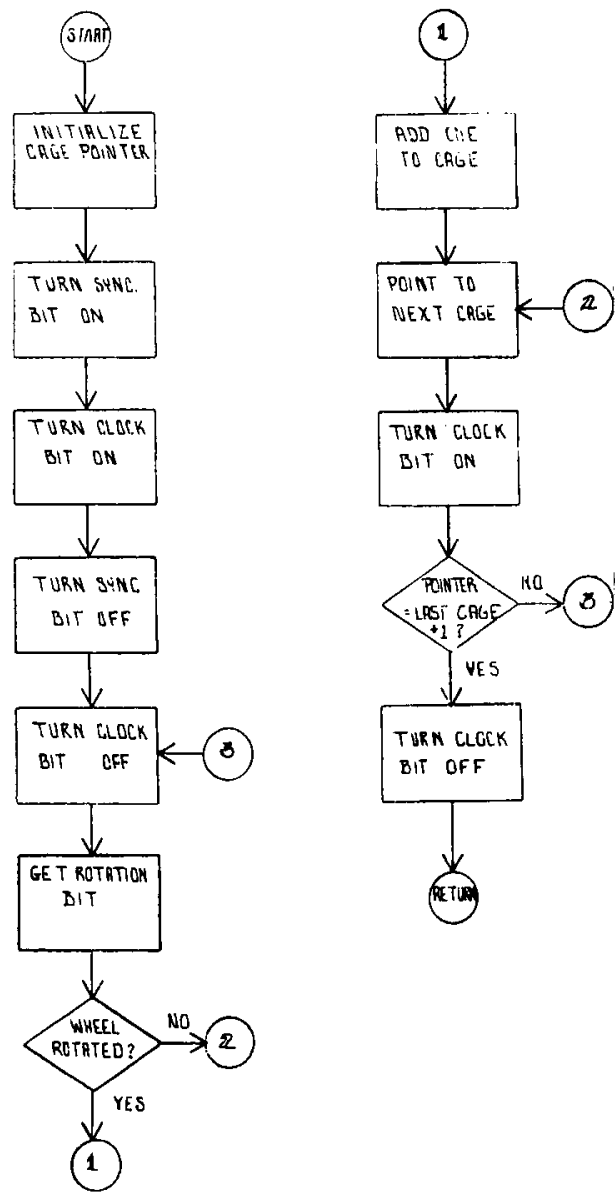

Figure 3. Algorithm for operation of interface card. to a different pin of the DB-25S connector. Six 22-pin connectors are tied together to form the basis of the motherboard. (See Figure 4.) The clock line is common to all cards, as is ground, $+5 \mathrm{~V}$, and master reset. Each card's data output is pulled up on the 22-pin edge connector by a 1-kohm resistor. Each card's data output is then applied to individual inputs of a NAND gate. Remembering that each card's data output is inverted, the result of the NAND gate output is a logic "OR" function of the noninverted data. A 7432 "ORs" the inputs from two NAND gates. A 2N2222 transistor acts as a buffer output and allows the motherboard to be "wired OR" by connecting motherboard data outputs together with a jumper. The half of the 7432 not used for data logic is wired as an R-S "NOR" gate flip-flop. A front panel switch connected to this flip-flop is used as master reset on start-up. No buffering of the clock pulses from the computer is necessary; in stacking devices, it would be.

The back panel of the motherboard has two DB-25S connectors. Each connector receives 24 lines of input from the real world.

The interface is designed so that the input (or data) lines connect to the common contacts of a microswitch. Ground is connected to the normally closed contact, and $+5 \mathrm{~V}$ are connected to the normally open contact. This arrangement permits ground and $+5 \mathrm{~V}$ to be "daisychained" from one switch to the other, and it requires that only the individual input lines from each wheel be run back to the motherboard. Input lines are run over $35 \mathrm{ft}$ with no noise problems.

For this application, the mechanical counters and

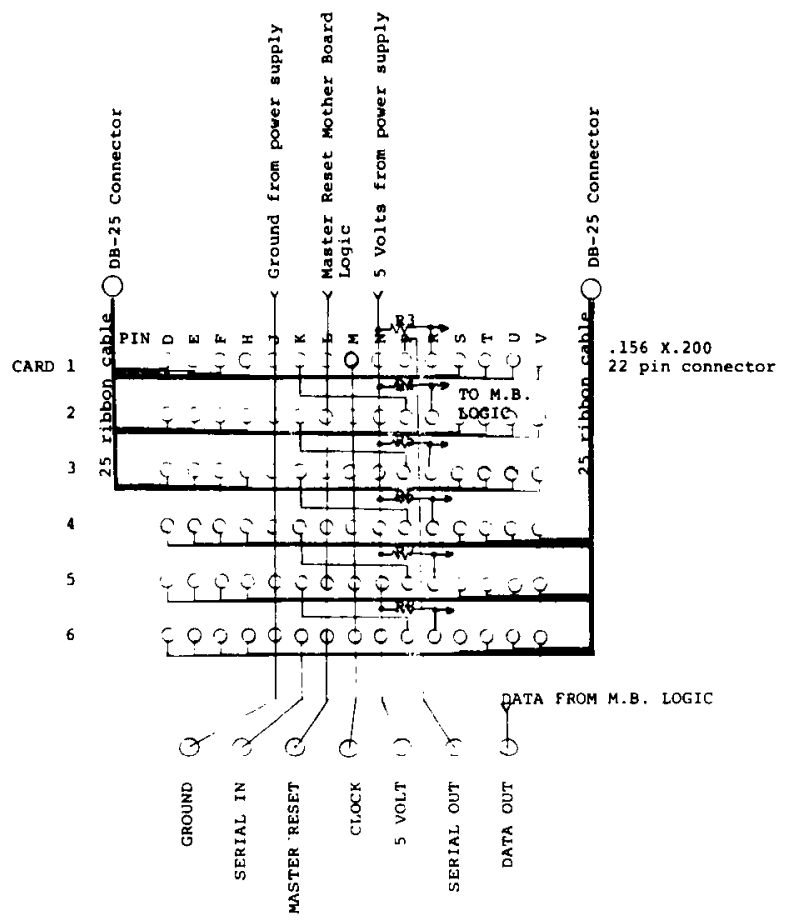

Figure 4. Motherboard circuitry. 
linkage on Wahmann LC.34 running wheels are replaced with a cam arrangement. The cam is threaded onto the shaft of the running wheel and a microswitch positioned so that the arm rests on the center portion of the cam. Thus, it requires a full revolution to operate the microswitch. A partial revolution or "rocking motion" results in "free-wheeling" on the shaft until sufficient distance is traveled to encounter a stop pin. At this point, the shaft turns the center portion and the microswitch closes, creating a pulse.

Software is written so that the wheels are polled approximately every $11 \mathrm{msec}$. If a revolution has occurred during the preceding interval, the appropriate counter for that animal is incremented. Since the interface is sensitive only to the leading edge of the pulse, on the next polling sequence a count will not be registered even if the switch is still closed. This design eliminates concern over such factors as simultaneous input pulses, duration of input pulses, and so on, which have to be dealt with in an interrupt-driven system.

Two other portions of the program (not described here) provide for cage-monitoring and data-dump routines. When the program is not polling the cages, it jumps to a monitor routine that allows an individual cage to be addressed and the counter containing the number of wheel revolutions to be examined. The data-dump routine allows the data collected over a set interval of time (from 1 to $24 \mathrm{~h}$ ) to be dumped to cassette tape for later retrieval and analysis.

The system has been used in studies up to 12 weeks in duration. The only significant problems encountered occurred when there was a building-wide power failure. Obvious improvements would be to put the software in EPROM, provide for automatic recovery in case of power failure, and/or implement a battery back-up feature. The system is flexible enough to be used in almost any situation that requires automatic data logging of a large number of inputs.

Complete schematics and PC board layouts for the system are available from the first author. Detailed drawings of the cam as well as a software listing of the program are also available.

\section{REFERENCE}

Osaka, N. An inexpensive microcomputer peripheral 1/O expansion. Behavior Research Methods \& Instrumentation, 1980, 12, 344-345. 